\title{
CHILE Y EL FORO ECONÓMICO ASIA-PACÍFICO APEC
}

\author{
Raul F. Campusano
}

Abogado. Profesor de Derecho Ambiental Internacional

Universidad Catolica del Norte (Sede Coquimbo)

Master of Arts, Notre Dame

Master en Derecho, Leiden

\section{1.- Introducción}

El Foro Económ. _o del Asia-Pacífico, APEC, es el mayor grupo regional del mundo actual. Representa a 2.100 millones de consumidores, $45 \%$ del PIB mundial, y tasas de ahorro interno entre 35 y 55\%. Al mismo tiempo, muestra rangos de inflación promedio de un dígito, un ingreso per cápita mediano a alto, o bajo pero con una gran población consumidora.

La misión de APEC tiene como principales objetivos apoyar el crecimiento y desarrollo de la región; contribuir al crecimiento y al desarrollo de la economía mundial: reforzar los efectos positivos resultantes de la creciente interdependencia económica, en la región y en la economía mundial; desarrollar un sistema comercial de multilateralismo abierto; y reducir las barreras al comercio de bienes, servicios e inversiones.

El objetivo central de APEC es alcanzar el libre comercio en el Asia-Pacífico, antes del año 2010 para las economías industrializadas (Japón, Corea, Estados Unidos, Australia), y antes del año 2020 para las no industrializadas. El esfuerzo se realiza a través de un activo programa de liberalización comercial consistente con la OMC, con dos mecanismos:

(i) la reciprocidad por parte de terceros países o regiones, y

(ii) la apertura unilateral de las economías de la región, en torno a la idea del unilateralismo concertado. Para facilitar los acuerdos, objetivos, y agenda de

\footnotetext{
'Actualmente, el Asia Pacifico es la región más dinámica del mundo entero, alcanzando el crecimiento más rá pido del comercio y la producción mundial, durante las últimas dos décadas. Tal dinamismo se ha traducido en un aumento cada vez más significativo y constante de su participación en el quehacer económico mundial. A diferencia de otras regiones, el auge comercial y la interdependencia económica en el Asia-Pacífico se han dado en gran medida por el empuje del sector privado, lo que ha significado una expansión acelerada del comercio de bienes y servicios y de la inversión directa, originando así uno de los mercados globales más importantes y activos de los últimos tiempos.
} 
desgravación, APEC impulsa extensos programas de facilitación del comercio e inversión, y de cooperación en campos de amplio espectro.

Durante este proceso, APEC sirve de foro consultivo intergubernamental para la discusión de distintos temas de cooperación regional. ${ }^{3}$ Su peso e importancia ha ido creciendo en la región, siendo ahora calificado como la organización más prometedora del Pacífico, y como la única entidad gubernamental que reúne "Economías" y no países, solucionando asi disyuntivas políticas. Lo que APEC busca, en su estado actual, es su transformación en una verdadera Comunidad Económica del Asia Pacífico en el mediano a largo plazo.

Desarrollo de la Regı́́sı

El proceso de vansformación económica que experimenta la economía mundial se asocia de manera importante al cambio de eje del dinamismo del crecimiento, desde las tradicionales potencias del Atlántico a la Cuenca del Pacífico. ${ }^{5}$ A pesar de los intentos integradores que se expresan en el concepto mismo de Cuenca del Pacífico, la región que así se denomina presenta una enorme diversidad cultural, de tamaño, y de realidades económicas, que es necesario tener en cuenta en cualquier análisis que se quiera emprender en esta materia y, en consecuencia, en cualquier estrategia que se diseñe.

Presente en toda la evolución de la cooperación trans-pacífica se encuentra la constatación de que en dicha área se concentran los países de economías más dinámicas del actual período histórico, a la vez que concentran la mayor densidad demográfica del planeta, lo que las convierte en mercados fundamentales para el crecimiento del comercio mundial. También es posible señalar, como un elemento del escenario que se ha mantenido en estos años, la persistencia de un modelo económico centrado fundamentalmente en el comercio exterior y las exportaciones. Aun cuando es posible detectar importantes diferencias en los caminos escogidos por los diferentes

\footnotetext{
${ }^{2}$ Ministerio de Relaciones Exteriores. Documento de trabajo interno, sin fecha.

${ }^{3}$ Ver: PEREZ, Marisol. Política Exterior de Chile hacia el Asia-Pacífico. En "Chile-Japón, un Siglo de Amistad". Comisión Chilena de Celebración del Centenario de las Relaciones Chile-Japón. Santiago, 1997.

"Ver: SUBERCASEAUX, Enrique. Chile y APEC 2004: Desafíos y Oportunidades ante Escenarios Cambiantes. En "Diplomacia". No 88, Julio-Septiembre 2001, Santiago.

${ }^{5}$ Para una relación extensa y sistemática ver: DOBBS-HIGGINSON. M S. Asia-Pacific, its Role in the World Disorder. The Japan Times Editor. Tokyo, 1993.También: Borthwick. Mark. Pacific Century: The Emergence of Modern Pacific Asia. Westview Press, Sydney, 1998. Desde una perspectiva diferente y ajena a la ciencia política, pero ilustradora, ver: CHU, CHIN-NING. The Asian Game. Stealth Productions Australia. St. Ives, 1995.

${ }^{5}$ Ministerio de Relaciones Exteriores. Documento de trabajo interno, sin fecha.
} 
países del área para realizar tal apertura, todos ellos han venido orientando sus economías hacia las exportaciones.

La evolución de la economía internacional en la década pasada y en la actual se ha caracterizado por la emergencia de dos realidades fundamentales: la primera es el proceso de interdependencia que ha sobrepasado las tradicionales fronteras nacionales y, la segunda, es la globalización, concepto que da cuenta del notable incremento en los flujos internacionales de capital y de los factores de producción a través de métodos de comunicación y transporte cada vez más eficientes. Este fenómeno que generalmente fue denominado internacionalización, y que se expresó en el incremento del comercio horizontal y vertical de unidades de producción fue particularmente significativo entre los países desarrollados en la década de los 80 .

Sin embargo, la globalización también comprende otra tendencia, aparentemente contradictoria con la internacionalización y es el reforzamiento del proceso de regionalización. Se refiere éste último, a la tendencia actual de los países a profitar de la proximidad geográfica de compartir ciertas metas globales, a la vez que fortalecen sus ventajas comparativas uniéndose en grupos regionales y subregionales para facilitar así el comercio y los vínculos comerciales que los unen. La congruencia de estas tendencias aparentemente contradictorias es la característica esencial del proceso de cooperación en el Pacífico y se reafirma una y otra vez en los documentos que dan cuenta de la evolución institucional de la región.

Una lectura frecuente de documentos originados en la región Asia-Pacífico permite afirmar que el concepto más utilizado cuando se describe el proceso de interdependencia regional es el de la cooperación, que tiene una connotación diversa a la que comúnmente se utiliza en nuestra región, y que describe principalmente formas de ayuda más o menos gratuita que aportan los países desarrollados a los países en desarrollo.

En el proceso de regionalización del Asia-Pacífico, el concepto de cooperación caracteriza el conjunto de políticas, estrategias y mecanismos que se diseñan para promover y fortalecer la interdependencia. La constatación de la diversidad cultural y de nivel económico, unida al convencimiento de las ventajas que conlleva la dependencia mutua, necesariamente exige que los países de la región elijan un camino sistemático y sostenido de cooperación fundado en objetivos claros y permanentes.

\footnotetext{
${ }^{7}$ Ministerio de Relaciones Exteriores. Documento de trabajo interno, sin fecha.

${ }^{8}$ BORA, BIJIT AND MARI PANGESTU, Eds. Priority Issues in Trade and Investment liberalization: Implications for the Asia-Pacific Region. Pacific Economic Cooperation Council, PECC. Singapore, 1996.

'BORTHWICK, MARK. PACIFIC CENTURY: The Emergence of Modern Pacific Asia. Westview Press, Sydney, 1998.
} 
Este enfoque de cooperación como un objetivo de creciente interdependencia a largo plazo, es el que explica la forma en que se ha venido conformando una "comunidad del Pacífico", un objetivo que debe resultar de aproximaciones sucesivas, que respetando la diversidad y la idiosincrasia de cada uno de los países involucrados, vaya conformando un espacio crecientemente interconectado, en el cual la flexibilidad, el consenso y la tolerancia son las únicas herramientas que pueden asegurar su efectiva materialización.

El incremento del comercio regional no ha estado, entonces, asociado al establecimiento de ventajas mutuas en el espacio regional, sino al aprovechamiento de los beneficios de la integración, vertical y horizontal y de las particularidades de cada país en su búsqueda de niveles crecientes de complementariedad. El enfoque de la integración económica no discriminatoria y de apertura de mercados que caracteriza a la región Asia-Pacífico es una propuesta de cooperación regional que postula el incremento del comercio como factor fundamental del dinamismo económico. La cooperación no puede ser, desde este punto de vista, una amenaza sino un aporte plenamente consistente con esta ampliación de los flujos comerciales globales. La filosofía económica dominante en la región ha sido el "regionalismo abierto" fundado en el establecimiento de un área de "cooperación" económica y comercial.

\section{2.- Evolución Institucional Regional}

Desde sus inicios, la idea de la integración entre las economías de los países de la región ha tenido diversas expresiones conceptuales. El enfoque predominante en el último tiempo se ha centrado fundamentalmente en reconocer como factor integrador el de la interdependencia económica y comercial de los actores ribereños de la Cuenca con la región Asia-Pacífico, que opera como centro dinamizador de este proceso y principal punto de origen de las iniciativas de institucionalización. Sin embargo, es interesante tener en cuenta que más allá de elaboraciones conceptuales predeterminadas, el proceso de institucionalización ha respondido a la necesidad de cooperación entre los distintos países del área. Ello explica que se haya venido transitando desde mecanismos de consulta empresarial o núcleos de relación académica, hasta el reciente establecimiento de una organización intergubernamental.

Este proceso se ha venido produciendo además, en los hechos, sin que el surgimiento de una nueva instancia implique una desaparición de instituciones previas que siguen existiendo y cumpliendo diversas funciones y articulando intereses para la persecución del objetivo último de todas ellas, que es el de acrecentar la cooperación entre los países de la Cuenca. Así, el proceso de creciente institucionalización de los

${ }^{10}$ Ministerio de Relaciones Exteriores. Documento de trabajo interno, sin fecha. 
países y regiones de la Cuenca del Pacífico que ha venido desarrollándose en las últimas décadas se ha realizado en torno al concepto de la "cooperación"."

Los principales promotores de los esquemas de integración en la Cuenca del Pacífico han sido Japón, Australia y Estados Unidos. A mediados de la década del sesenta surgen los primeros proyectos de gestación de un área de libre comercio en la región. En noviembre de 1965, el profesor japonés Kiyoshi Kojima de la Universidad Hitotsubashi, planteó una zona de libre comercio del Pacífico (AFTA: Pacific Free Trade Area), que consistía en el establecimiento de un área comercial entre Estados Unidos, Canadá, Japón, Australia y Nueva Zelandia, cuya acción económica se irradiaría al resto de la región y serviría de apoyo al desarrollo de las naciones asiáticas.

En el campo empresarial, en el año 1967, el sector privado estableció el Consejo Económico de la Cuenca del Pacífico (PBEC: Pacific Basin Economic Council). Organismo de encuentro, contacto y coordinación entre los empresarios de las naciones de la región. EI PBEC surge como una organización no gubernamental de hombres de negocio de la Cuenca del Pacífico. Aún cuando opera bajo auspicios privados, mantiene relaciones directas con los gobiernos regionales y con diversas agencias internacionales. Se ha constituido en un activo líder para la comunidad empresarial de la zona. Sus principales objetivos han sido fomentar los beneficios mutuos de la cooperación económica y del progreso social de toda la Cuenca del Pacífico. Se ha tratado así, de mejorar todo lo referido a los negocios empresariales, robustecer el sistema de empresa privada, generar oportunidades de negocios, incrementar el comercio y la inversión en la zona.

EI PBEC persigue sus propósitos a través de dos vías principales; una es servir de foro internacional para el intercambio de ideas, opiniones y experiencias y la otra es proporcionar consejo y asesoría a gobiernos y agencias internacionales sobre economía y negocios relacionados a la Cuenca del Pacífico. Las empresas y empresarios miembros se afilian al PBEC Internacional a través de comités nacionales. Cada comité miembro tiene un Director General, normalmente perteneciente a una institución empresarial que se vincula directamente con la Secretaría Internacional. Además existen comités especiales, creados para enfrentar temas específicos.

\footnotetext{
${ }^{11}$ Ministerio de Relaciones Exteriores. Documento de trabajo interno, sin fecha.

${ }^{12}$ Esta idea junto con varias otras han sido promotoras del concepto APEC, pero también debe considerarse que varias de ellas tenian en su núcleo concepciones distintas de integración, como por ejemplo excluir a los países del continente americano, y, en algunos casos, incluso a Australia y Nueva Zelandia. Lo mismo puede decirse en cuanto a su concepción filosófica y política, e incluso económica. También debe considerarse que el tema China siempre cruzó todas estas propuestas complicándolas en su concresión práctica. Para ver contexto: DobbsHigginson, M. S. Asia-Pacific, its Role in the World Disorder. The Japan Times Editor. Tokyo, 1993.
} 
Posteriormente, en enero de 1968, el mismo Kojima junto al académico australiano Peter Drysdale de la Australian National University, expusieron un proyecto sobre una Organización para el Comercio y el Desarrollo del Pacífico (OPTAD: Organization of Pacific Trade and Development). Casi una década más tarde, en diciembre de 1977, Jiro Tokuyama y el Nomura Research Institute de Japón, dieron a conocer una propuesta sobre cooperación económica en el Pacífico. Esta iniciativa tuvo un carácter más bien funcional, pues la idea era concentrar los esfuerzos globales en la creación de un acuerdo zonal relativo al desarrollo de proyectos de energía y producción de acero, junto con la creación de un Fondo de Cooperación. ${ }^{13}$

En 1978, los planteamientos académicos en gestación se enriquecen con la idea de crear programas de investigación centrados en el estudio de la interdependencia económica de los países de la región; orientados, especialmente, a la capacitación de dirigentes empresariales. Así surgió el Pacific Rim Project, auspiciado por la Universidad de Washington. El año 1979 el Korean International Economic Institute desarrolla un importante estudio sobre la idea de una Comunidad Pan-Pacífica; realizado desde el punto de vista de la tendencia integradora observada en la región durante los últimos años. ${ }^{14}$ El mismo año acadé micos japoneses sugieren la creación de una Comisión del Pacífico, una organización consultora a nivel privado, destinada a establecer vínculos entre los países industrializados y los países en vías de desarrollo. ${ }^{15}$

Ya en la década de los ochenta, el Consejo Económico de la Cuenca del Pacífico (PBEC) se plantea el objetivo de asesorar a gobiernos y agencias gubernamentales internacionales en materias de cooperación económica, originando el concepto de "Cooperación en la Cuenca del Pacífico" (Pacific Basin Cooperation Concept) de cuyo desarrollo surge uno de los estudios más completos realizados hasta aquel momento. En él se promocionaba la creación de un organismo privado destinado a canalizar las iniciativas de cooperación económica al interior de la región, sobre la base del libre comercio y la coordinación y complementación con los acuerdos bilaterales y multilaterales ya existentes. De esta manera, a partir de los ochenta, comienzan a hacerse realidad los proyectos institucionales de integración de las décadas anteriores. En tal contexto, la gran iniciativa de esta década, fuertemente marcada por el concepto de "Cooperación", fue la PECC (Pacific Economic Cooperation Conference), surgida sobre la base del extraordinario crecimiento económico y la cada vez mayor interdependencia de las economías de la región.

\footnotetext{
${ }^{13}$ Ministerio de Relaciones Exteriores. Documento de trabajo interno, sin fecha.

${ }^{14}$ BORTHWICK, Mark. Pacific Century: The Emergence of Modern Pacific Asia. Westview Press, Sydney, 1998.

${ }^{15}$ BORTHWICK, Mark. Pacific Century: The Emergence of Modern Pacific Asia. Westview Press, Sydney, 1998.
} 
EI PECC nace de una iniciativa del primer ministro japonés Massayoshi Ohira y el Premier australiano Malcom Frazer, quienes coincidieron en la importancia de darle trascendencia al desarrollo del concepto de Cooperación en la Cuenca del Pacífico y convocaron a un seminario internacional sobre la materia. Esta primera conferencia se llevó a cabo en la Universidad Nacional de Australia, en Camberra, y contó con la participación de empresarios académicos y representantes del sector público de quince países del Pacífico. Con el tiempo, la iniciativa se estructuró como una conferencia anual tripartita con representantes del sector empresarial, gubernamental y académico. Luego del tercer encuentro, este ejercicio de reuniones informales adoptó una estructura formal y comenzó a denominarse Pacific Economic Cooperation Conference.

Los principales objetivos de estas reuniones son identificar y coordinar la acción, el pensamiento y las iniciativas de los tres estamentos; facilitar e incrementar la cooperación económica entre los países de la Cuenca del Pacífico; así como también crear los mecanismos necesarios para la materialización de dichos objetivos, fomentando políticas a mediano y a largo plazo, orientadas a configurar esquemas de cooperación económica apropiados para constituir las bases de una futura comunidad del Pacífico (el trabajo del PECC se desarrolla a través de su Conferencia, que se reúne cada doce o dieciocho meses; el Comité Permanente y diversos Grupos de Trabajo). ${ }^{17}$

Como una de las iniciativas más importantes de este período de fines de la década, el año 1989, a partir de una propuesta del entonces primer ministro australiano Bob Hawke, se crea el Asia Pacific Economic Cooperation (APEC) como un foro de Ministros y Altos Funcionarios de Gobierno de los países de la Cuenca del Pacífico capaz de generar un esquema de cooperación intergubernamental basado en lazos formales. Así, la creación de APEC representa un paso fundamental como el primer esfuerzo serio, a nivel gubernamental, para la generación de una instancia de cooperación económica, y determina significativamente el quehacer económico de la región para la década de los noventa ${ }^{18}$.

\section{3.- Conceptos introductorios sobre APEC}

En su esencia, APEC es un foro consultivo intergubernamental de carácter informal de la región Asia-Pacífico, conformado por las economías de dieciocho países.

\footnotetext{
${ }^{16}$ Ver: PEREZ, Marisol. Política Exterior de Chile hacia el Asia-Pacífico. En Chile-Japón, un Siglo de Amistad. Comisión Chilena de Celebración del Centenario de las Relaciones Chile-Japón. Santiago, 1997.

Ministerio de Relaciones Exteriores. Documento de trabajo interno, sin fecha.

${ }^{19}$ Ver: Dirección Asia-Pacífico, Ministerio de Relaciones Exteriores. Participación de Chile en los Organismos de Cooperación Transpacífica. Sitio web del Ministerio.
} 
Fue creado por una iniciativa del Gobierno australiano el año 1989 y sus principales objetivos son:

$>$ Apoyar el crecimiento y el desarrollo de la región;

$>$ Contribuir al crecimiento y al desarrollo de la economía mundial;

> Reforzar los efectos positivos, tanto para la región como para la economía mundial, resultantes de la creciente interdependencia económica;

$>$ Desarrollar un sistema comercial de multilateralismo abierto, y

$>$ Reducir las barreras al comercio de bienes y servicios y favorecer las inversiones recíprocas.

El cumplimiento de los objetivos antes expuestos se desarrolla en el marco de los siguientes principios:

$>$ Adhesión a los principios de la libertad y el libre mercado como marcos del desarrollo económico

$>$ Toma de decisiones por consenso.

$>$ "Economías" y no países.

$>$ Conformidad con los acuerdos GATT-OMC;

$>$ Liberalización y facilitación del comercio y las inversiones;

$>$ Regionalismo abierto; $^{19}$

> Facilitación y favorecimiento de la cooperación económico-técnica;

$>$ Reconocimiento de los distintos estados de desarrollo y diversidad cultural de los miembros de APEC. ${ }^{20}$

Los veintiún países (llamados dentro de este contexto "Economías")que conforman APEC son:

Australia

\footnotetext{
${ }^{19}$ Ver: Dirección Asia-Pacífico, Ministerio de Relaciones Exteriores. Participación de Chile en los Organismos de Cooperación Transpacífica. Sitio web del Ministerio. También ver: Dirección Asia-Pacífico, Ministerio de Relaciones Exteriores. Relaciones Politicas y Económicas Bilaterales. Sitio web del Ministerio 20
} 
Nueva Zelandia

Los seis países del ASEAN: 1-Brunei Darussalam; 2-Filipinas; 3-Indonesia; 4Malasia; 5-Singapur; 6-Tailandia

Japón

Corea

Las tres Chinas: 1-República Popular China; 2-Taiwan; 3-Hong-Kong

Canadá

$>$ Estados Unidos

$>$ Papua Nueva Guinea

\section{México}

Chile

Perú

\section{Rusia}

\section{Vietnam}

Con el reintegro de Hong-Kong a la República Popular China se produjo una situación interesante en relación con el tema de la representación de esa Economía, pero que refleja la especial naturaleza de la asociación dentro de APEC.'

APEC es un foro de ministros y autoridades de gobierno, que ha logrado instituirse como una entidad intergubernamental que reúne economías más que a países. $^{22}$ Su funcionamiento se lleva a cabo sobre la base de reuniones anuales en el ámbito ministerial, realizadas fundamentalmente con los ministros de Economía y de Relaciones Exteriores de los países miembros, y reuniones de altos funcionarios de gobierno, organizadas sobre la base de Grupos de Trabajo. La idea ha sido crear una organización dinámica y eficiente, que no requiera de estructuras rígidas para operar,

\footnotetext{
Primeramente, ver el excelente trabajo: WILHELMY, Manfred. El proceso de Reformas en China y su Política Exterior. Estudios Públicos, Centro de Estudios Públicos, N 78, 2000. También ver: BURSTEIN, Daniel y Arne de Keijzer. Big Dragon: China's Future, what it means for Business, the Economy, and the Global Order. New York, 1998. También ver: TORO, Sergio. 2001 Annus Admirabilis: La Política Exterior de China de la Guerra del Opio a la Globalización. En Diplomacia. N 88, Julio-Septiembre 2001, Santiago.

${ }^{22}$ Ver: Dirección Asia-Pacífico, Ministerio de Relaciones Exteriores. Participación de Chile en los Organismos de Cooperación Transpacífica. Sitio web del Ministerio.
} 
pues existe la convicción de que, a través del establecimiento de un sistema flexible, se podrá contar con una participación más activa y enriquecedora de los países miembros. De esta manera, uno de los aspectos más relevantes de APEC es su carácter informal (en términos estrictos, APEC no constituye un tratado internacional). ${ }^{23} \mathrm{La}$ organización de APEC se construye buscando la eficacia y la eficiencia en el logro de los objetivos de desarrollo e integración del Asia-Pacífico y en un quehacer dinámico y flexible, manifestado en un sistema operativo estructurado en torno a las siguientes instancias:

\section{> Reunión de Líderes (AELM)}

Reúne, una vez al año, a los Jefes de Estado de las Naciones miembros, con el fin de delinear las políticas globales de APEC. La primera de estas reuniones tuvo lugar en Seattle, U.S.A. en el mes de noviembre del año 1993, inmediatamente después de la quinta Reunión Ministerial (realizada también en Seattle). En ella los líderes de los países miembros acordaron reunirse en el futuro aprovechando la instancia de las Reuniones Ministeriales.

Este primer encuentro de Jefes de Estado constituyó una excelente oportunidad para que los líderes de los países de APEC compartieran sus puntos de vista respecto del desarrollo futuro de la Organización y comenzaran a desarrollar un mayor sentido de comunidad. Además, en esta reunión, se reconoció la necesidad de acelerar el proceso de liberalización del intercambio comercial y el flujo de inversiones recíprocas, y se acordaron medidas específicas para el logro de tales fines. El segundo encuentro de Jefes de Estado se realizó el mes de noviembre del año 1994 en Indonesia y el próximo se realizará en Japón a fines de este año (1995). La idea de estas Reuniones de Líderes es generar una instancia informal en la cual los Jefes de Estado de los países miembros puedan compartir sus puntos de vista respecto del desarrollo y evolución de APEC.

\section{$>$ Reuniones Ministeriales (MM)}

Al menos una vez por año, se reúnen los Ministros de Relaciones Exteriores y Economía de los distintos países para evaluar, diseñar y poner en práctica las respectivas políticas gubernamentales vinculadas a APEC. En estas reuniones se toman decisiones de manejo, estructura institucional y membrecía; se aprueba el plan de manejo y el presupuesto futuros, $y$, en general, se determinan las pautas direccionales para el año siguiente. La sede de los encuentros va rotando año a año entre los países miembros. El primero de estos encuentros se realizó el mes de noviembre del año 1989 en la ciudad de Camberra, Australia y luego, sucesivamente; el año 1990 en Singapore, el año 1991 en Seoul, el año 1992 en Bangkok, el año 1993 en Seattle, el año 1994 en

Ministerio de Relaciones Exteriores. Documento de trabajo interno, sin fecha. 
Bogor, el año 1995 en Osaka, Japón, el año 1996 en Filipinas y el año 1997 en Canadá.

A la primera reunión ministerial, realizada en Camberra, asistieron 26 Ministros pertenecientes a doce países de la región (Australia, Brunei, Canadá, Indonesia, Japón, Corea, Malasia, Nueva Zelandia, Filipinas, Singapur, Tailandia y Estados Unidos) y se aceptó la concurrencia de tres organizaciones como observadores oficiales: La Asociación de Naciones del sudeste Asiático (ASEAN), El Consejo de Cooperación Económico del Pacífico (PECC) y El Foro del Pacífico Sur (SPF). En esta reunión los Ministros acordaron los principios básicos de APEC y determinaron las pautas de desarrollo de la organización.

Además de las reuniones antes mencionadas, se llevan a cabo una serie de reuniones de Ministros de otras carteras. Así, por ejemplo, el año 1992 hubo una reunión de Ministros de Educación de los países APEC en la ciudad de Washington D.C. y en el año 1994, una reunión de Ministros de Finanzas en el mes de febrero y una de Ministros de Medio Ambiente en el mes de abril.

Reuniones de funcionarios de alto nivel (SOM)

Cada país miembro de APEC debe nombrar un representante de alto nivel de su Cancillería como "Senior Official Member" (SOM); el cual, junto con ser el representante del país, es el responsable de coordinar las actividades de todos los sectores que representen a su economía ante APEC. Dichos funcionarios se reúnen frecuentemente a lo largo del año, entre una Reunión Ministerial y otra. A diferencia de organismos tales como las Naciones Unidas o la Organización Mundial del Comercio, APEC no tiene una instancia institucional con sede determinada, y por ello las reuniones SOM constituyen "la oportunidad de implementación" de las decisiones Ministeriales; además de ofrecer un apoyo subsidiario fundamental a los Comités y Grupos de Trabajo.

\section{$>$ Grupo de personas Eminentes (EPG)}

Son los encargados de asesorar a los Líderes, Ministros y SOMs en la formulación de las políticas de APEC en el marco del desarrollo futuro de la región del Asia-Pacífico. El Grupo nace de una iniciativa presentada por los representantes australianos en la cuarta Reunión Ministerial (MM) de Bangkok, con el objetivo de originar una instancia de estudio y análisis del desenvolvimiento económico de la

\footnotetext{
${ }^{24}$ Ver: Dirección Asia-Pacífico, Ministerio de Relaciones Exteriores. Participación de Chile en los Organismos de Cooperación Transpacífica. Sitio web del Ministerio.

${ }^{25}$ Ministerio de Relaciones Exteriores. Documento de trabajo interno, sin fecha.
} 
región en el largo plazo. Se encuentra conformado por distinguidos académicos, hombres de negocio, políticos y ex-funcionarios de Gobierno de los diversos países y economías de APEC.

El primer informe del Grupo, titulado "A Vision for APEC: Towards an Asia Pacific Economic Community", fue entregado en la Reunión Ministerial (MM) de Seattle del año 1993. En dicho informe se recomendó a los líderes de APEC tomar iniciativas respecto de cuatro grandes áreas: Liberalización del intercambio global y regional, programas de facilitación del intercambio, cooperación técnica y la institucionalización organizativa de APEC. El segundo informe del Grupo, titulado: "Achieving the APEC Vision: Free and Open Trade in the Asia Pacific, fue entregado en Indonesia el año 1994 en la sexta Reunión Ministerial (MM) y se centró en la liberalización del intercambio y su relevancia económica para APEC.

\section{$>$ Grupos especiales o Comités}

Comité de comercio e inversión (CTI): Este grupo fue creado el año $19 \overline{9} 3$ y su labor fue fijada por un acuerdo marco establecido en la Reunión Ministerial (MM) de Seattle. En él, se estructuran las políticas futuras de facilitación del intercambio y flujo de inversiones de la región y se tratan los temas prioritarios vinculados a la liberalización comercial, al flujo de capitales e inversión, y el comercio de bienes, servicios y tecnología.

Grupo ad-hoc en tendencias económicas (ETI): Este grupo fue creado el año 1991 con la finalidad de revisar los más amplios aspectos económicos regionales, determinar la naturaleza de la interacción económica de la región e identificar los grandes desafíos económicos para el corto y mediano plazo. De esta manera, el Comité es el encargado de recopilar y entregar los antecedentes de carácter técnico relativos a la situación económica dentro de la región del Asia-Pacífico.

Comité de administración y presupuesto (BAC): Este comité fue creado para asesorar al SOM en materias administrativas y de presupuesto con respecto al programa de trabajo de APEC.

$>$ Grupos de trabajo:

Además de los organismos señalados anteriormente, existen los siguientes grupos de trabajo encargados de desarrollar labores específicas:

\footnotetext{
${ }^{26}$ Ver: Dirección Asia-Pacífico, Ministerio de Relaciones Exteriores. Participación de Chile en los Organismos de Cooperación Transpacífica. Sitio web del Ministerio.

${ }^{27}$ Ministerio de Relaciones Exteriores. Documento de trabajo interno, sin fecha.
} 
1. Datos de comercio e inversión:

2. Promoción de exportaciones;

3. Recursos marinos;

4. Pesca;

5. Recursos humanos;

6. Telecomunicaciones;

7. Transporte;

8. Turismo;

9. Ciencia y tecnología y

10. Cooperación energética.

\section{Secretaría Administrativa}

Es el órgano administrativo de APEC encargado de velar por el funcionamiento de la organización, manejar su presupuesto, coordinar el desarrollo de los programas de trabajo y servir como centro de información. Es encabezada por un director ejecutivo, cuyo cargo dura un año.

\section{Una red de Empresarios del Asia Pacífico:}

(Asia Pacific Business Forum) Conforma un foro que representa la voz empresarial al interior de APEC.

\section{4.- Consideraciones Históricas de APEC}

La primera observación que surge al analizar la región del Asia-Pacífico es que ésta ha experimentado un gran dinamismo económico y una creciente interdependencia. ${ }^{29}$ Sin embargo, como resultado de los problemas estructurales y la naturaleza cada vez más compleja de esta interdependencia, en los últimos años han

\footnotetext{
${ }^{28}$ Es relevante notar que el Grupo de Trabajo de Cooperación Energética es el que aprobó la creación del Grupo Experto en minería, exploración y desarrollo Energético, GEMEED-APEC, cuya sede está en Chile y cuyo presidente, Tomás Astorga, es chileno. Sobre esta organización se detalla más adelante.

${ }^{29}$ Ver: SUBERCASEAUX, Enrique. Chile y APEC 2004: Desafios y Oportunidades ante Escenarios Cambiantes. En Diplomacia. $N^{\circ}$ 88, Julio-Septiembre 2001, Santiago. También: ARTAZA, Mario Ignacio. Chile en APEC: Aprovechando lo mejor de la Globalización. En Diplomacia. N88, Julio-Septiembre 2001, Santiago.
} 
surgido grandes presiones e incertidumbres. Los desbalances comerciales en la zona aumentaron a niveles alarmantes y llevaron a serias tensiones comerciales entre el Este Asiático y los Estados Unidos. El aumento del proteccionismo, la tendencia a la formación de acuerdos comerciales bilaterales o regionales y los desajustes en el marco estructural del GATT, fueron creando temor respecto de las perspectivas de crecimiento para la región.

La situación descrita originó preocupación al interior del área y propició diversos llamados para algún tipo de acción colectiva. A partir de 1988, se registró una proliferación de nuevas propuestas e iniciativas para un diálogo y una cooperación regional más cercanos. De esta manera, en enero de 1989 en Seúl, Corea, se reunieron Ministro de Relaciones Exteriores del área para analizar temas relativos al sistema comercial y el desarrollo de la zona. En esa ocasión, el Primer Ministro australiano Bob Hawk, lanzó la propuesta para establecer un esquema de cooperación en la Cuenca del Pacífico basado en lazos intergubernamentales formales, lo que más tarde sería APEC (Asia Pacific Economic Cooperation) ${ }^{31}$. La iniciativa llamaba a establecer los mecanismos que permitiesen al análisis de temas económicos y sociales para que fuesen considerados en las formulaciones de políticas de desarrollo de los gobiernos respectivos. El objetivo era poner nuevamente en marcha la cooperación económica regional y al mismo tiempo establecer las bases de una respuesta del Pacífico asiático a las amenazas proteccionistas en el comercio exterior. Esta propuesta, que se centraba exclusivamente en Asia, suscitó gran interés y debate.

Por otra parte, la exclusión de los países de América del Norte del "grupo medular" de la organización, motivó que Japón reaccionara con cautela ${ }^{32}$. Por su parte, los miembros de ASEAN, se preocuparon frente a la posibilidad de que la propuesta debilitara considerablemente el marco de referencia ya establecido por ASEAN mediante el proceso de diálogo que lo vinculaba regularmente con los principales países con los que mantenía relaciones comerciales. Tampoco estaban de acuerdo con la creación de estructuras institucionales formales que pudieran encaminarse hacia el establecimiento de un bloque comercial regional, que terminaría por causar el colapso del sistema de comercio multilateral consagrado en las normas del GATT.

Finalmente, la postura generalizada a favor de la participación de Estados Unidos y Canadá adoptada por los países de ASEAN y Japón, así como las presiones del Gobierno de Estados Unidos, llevaron a Australia a examinar nuevamente su

\footnotetext{
${ }^{30}$ Ministerio de Relaciones Exteriores. Documento de trabajo interno, sin fecha.

${ }^{31}$ Ver: BORTHWICK, Mark. Pacific Century: The Emergence of Modern Pacific Asia. Westview Press, Sydney, 1998.

${ }^{32}$ Ver por contexto: SMITH. Patrick. Japan, a Reinterpretation. Vintage Books, New York, 1997.
} 
proyecto de cooperación y a ampliar el número propuesto de sus miembros. En la conferencia post-ministerial de ASEAN de julio de 1989, se llegó a consenso sobre la asistencia a una reunión ministerial exploratoria que se celebraría en Camberra, Australia, en noviembre de 1989, con el objeto de analizar la cooperación en el Pacífico asiático con Canadá, Japón, Nueva Zelandia, Corea del Sur y Estados Unidos. Se indico que la República Popular China, Hong Kong y Taiwán no podía ser invitados a participar para evitar controversias. ${ }^{33}$ Comenzó, entonces, un intenso proceso de consultas entre Australia y otros 11 países del Asia-Pacífico, que llevó nueve meses después a la Primera Junta Ministerial de APEC en Camberra. Esta fue presidida por el Ministro australiano de Relaciones Exteriores y Comercio, Senador Gareth Evans. A esta reunión asistieron 26 Ministros de los Gobiernos de Australia, Brunei, Canadá, Corea, Estados Unidos, Filipinas, Indonesia, Japón, Malasia, Nueva Zelandia, Singapur y Tailandia.

La formación de APEC representó un paso importante como el primer esfuerzo serio, a nivel gubernamental, hacia una cooperación económica en la región. Se puede afirmar que ésta se construyyó y alimentó a partir de un trabajo base indispensable, derivado especialmente del PBEC y del PECC, que han hecho una contribución significativa para despertar una conciencia regional de que existen intereses comunes que pueden promoverse a través de una mayor consulta y cooperación. En la conferencia hubo consenso sobre los principios generales que guiarían la creación de un sistema multilateral abierto de comercio, destinado a sostener el crecimiento y desarrollo de la región, reconociendo su diversidad y basando la cooperación en el intercambio informal de consultas. Además, se explicitó la intención que la cooperación no implicaría la formación de un bloque comercial, sino que ésta debería fortalecer las ventajas de la interdependencia a nivel regional y mundial. De esta forma, se dejaba en claro la necesidad de la región del Este de Asia de tener acceso a los mercados de Occidente para continuar su desarrollo ${ }^{34}$.

\section{5.- Evolución de APEC}

Se han efectuado numerosas Reuniones Ministeriales de APEC hasta la fecha. Al observar la evolución y revisar el temario de estas conferencias, se puede apreciar que el tema principal y permanente ha sido la liberalización comercial no discriminatoria o regionalismo abierto, como una alternativa para evitar la formación de bloques

\footnotetext{
${ }^{33}$ Ver por contexto: WILHELMY, Manfred. El proceso de Reformas en China y su Política Exterior. Estudios Públicos, Centro de Estudios Públicos, N 78, 2000. También ver: BURSTEIN, Daniel y ARNE DE KEIJZER. Big Dragon: China's Future, what it means for Business, the Economy, and the Global Order. New York, 1998. también ver: TORO, Sergio. 2001 Annus Admirabilis: La Política Exterior de China de la Guerra del Opio a la Globalización. En Diplomacia. N88, Julio-Septiembre 2001, Santiago.

Ministerio de Relaciones Exteriores. Documento de trabajo interno, sin fecha.
} 
económicos cerrados. A este respecto se han distinguido diferentes posiciones dentro del organismo. Por un lado, Estados Unidos, Japón. Australia y Canadá, han sostenido con mayor énfasis el comercio multilateral libre. Por el otro, Malasia ha propiciado la creación de un sistema más restringido que proteja a los países de ASEAN de los intereses de las grandes potencias del área.

La liberalización comercial y la inversión regional son temas centrales, ya que constituyen una de las razones de ser APEC y son una fuerza gravitante del desarrollo integral de la economía regional a través de un proceso de especialización vertical y horizontal de las industrias, de acuerdo con las cambiantes ventajas comparativas de la región. Se puede advertir que en el período inicial de APEC se dio importancia a la definición de los objetivos y principios generales del mecanismo, como forma de establecer un consenso y un marco de trabajo que guiara la organización ${ }^{35}$.

La estructuración, los mecanismos de administración institucional y aquellos relacionados con presupuesto también han constituido un asunto fundamental de discusión. Aquí también han existido distintas percepciones al no haber consenso en cuanto a qué forma de organización era la más adecuada. Otro de los temas prioritarios ha sido el ingreso de nuevos miembros, como fue el caso de la incorporación de las tres Chinas. ${ }^{36}$ Algunos de los integrantes de APEC eran partidarios de una moratoria hasta que no se fortalecieron institucionalmente. Otros, pretendían obstaculizar la incorporación de países no asiáticos, en especial los latinoamericanos, ya que no deseaban la eventual formación de un bloque latinoamericano que debilitara la posición de la presencia asiática al interior del organismo o que estos países rivalizaron con las economías más débiles del Asia-Pacífico en el largo plazo ${ }^{37}$.

En la medida que APEC se ha ido consolidando en el tiempo y tomando en cuenta las necesidades que han ido surgiendo dentro de ella, en las reuniones los Ministros han acordado la creación de diversas instancias para facilitar y hacer más efectivo el trabajo, ya sea estableciendo nuevos grupos y comités, impulsando iniciativas de trabajo y propuestas concretas para facilitar el comercio, etc. Otra materia fundamental que se ha tratado es la necesidad de coordinar las políticas económicas con el fin de que los países estén preparados para enfrentar los cambios que ocurran. En este sentido, se ha manifestado que APEC puede sugerir estrategias

\footnotetext{
${ }^{35}$ Ver: Dirección Asia-Pacífico, Ministerio de Relaciones Exteriores. Participación de Chile en los Organismos de Cooperación Transpacífica. Sitio web del Ministerio.

${ }^{36}$ BURSTEIN, Daniel y Arne de Keijzer. Big Dragon: China's Future, what it means for Business, the Economy, and the Global Order. New York, 1998. También ver: TORO, Sergio, 2001 Annus Admirabilis: La Política Exterior de China de la Guerra del Opio a la Globalización. En Diplomacia. N 88, Julio-Septiembre 2001. Santiago.

${ }^{37}$ Ministerio de Relaciones Exteriores. Documento de trabajo interno, sin fecha.
} 
comunes sobre aspectos económicos relevantes para la región. Finalmente, en las últimas reuniones se ha hecho especial referencia a la importancia del sector empresarial/privado en el área y la necesidad de aumentar los vínculos de este ámbito con APEC, incorporándolo a los Grupos de Trabajo, solicitando su asesoría y ampliando la cooperación ${ }^{38}$.

Todo parece indicar que la región del Asia-Pacífico se dirige hacia un modelo multilateral de desarrollo, en el cual se respeten las diferencias individuales, pero destacando al mismo tiempo aquellos aspectos complementarios y tomando en cuenta las ventajas comparativas de cada nación. Lo anterior se ve reforzado por los resultados positivos que han alcanzado en sus niveles de crecimiento económico. Los principios generales que guian a APEC reflejan claramente que ese es el camino que se desea que recorran estas economías. Su rápido éxito se debe en gran medida a la confianza que tiene en la apertura de la región. La existencia de este mecanismo ha sido un poderoso voto de confianza de sus miembros en el futuro de un sistema de comercio internacional liberal y justo, y una refutación a los que pronosticaban un mundo dominado por bloques de comercio discriminatorios, basados en Japón, Estados Unidos y la Comunidad Económica Europea. Con el tiempo, APEC podría evolucionar hacia acuerdos de libre comercio de toda la región, englobando a todos los acuerdos subregionales existentes. Esto le permitiría, junto con la Comunidad Económica Europea y el NAFTA, servir como uno de los pilares regionales que apoya y fortalece al sistema de libre comercio multilateral ${ }^{39}$.

En relación con PECC, el esquema original de esta organización ha quedado algo obsoleto ante la magnitud de las transformaciones, experimentadas en la región y en el mundo. De esta forma, el centro de gravedad de las consultas ha tendido naturalmente a desplazarse hacia APEC, con ocasión de las reuniones de representantes gubernamentales. En la actualidad, las Fuerzas de Tarea de PECC se están convirtiendo de facto en la antesala de las reuniones ministeriales, elaborando informes y proposiciones que refuerzan las negociaciones intergubernamentales en el seno de APEC. PECC seguirá siendo, sin lugar a dudas, un mecanismo valioso como instancia de comunicación intergubernamental, empresarial y académica, y especialmente como vía de comunicación y de inserción de América Latina en la Cuenca del Pacífico, pero dispondrá de un peso político limitado.

\footnotetext{
${ }^{38}$ Ver: Dirección Asia-Pacífico, Ministerio de Relaciones Exteriores. Participación de Chile en los Organismos de Cooperación Transpacífica. Sitio web del Ministerio.

${ }^{39}$ Ver: Dirección Asia-Pacífico, Ministerio de Relaciones Exteriores. Relaciones Políticas y Económicas Bilaterales. Sitio web del Ministerio.

${ }^{40}$ Ministerio de Relaciones Exteriores. Documento de trabajo interno, sin fecha.
} 
En los últimos años han surgido distintas vías de acercamiento entre las actividades de PECC y APEC. Los integrantes del PECC están interesados en lograr una mayor coordinación con los de APEC, para tener una mayor influencia en los ámbitos de decisión gubernamentales relacionados con la cooperación regional. Es bastante probable que a futuro ambas organizaciones jueguen un rol complementario, aprovechando el carácter tripartito del PECC, su nivel de estructuración y, por otra parte, la creciente gravitación de APEC como foro político. ${ }^{41}$ Posiblemente el PECC se transforme a la larga en una instancia especializada en materias técnicas de APEC. A su vez ésta se transformará en el órgano fundamental de la cooperación transpacífica, por su significativo peso político debido a la activa participación en su interior a. las potencias económicas del área, la tres Chinas, y las economías emergentes dei AsiaPacífico. Por otra parte, APEC debiera evolucionar de la etapa de construcción institucional hacia una orientada a la formulación de políticas. La liberación del comercio regional y el avance de los proyectos de trabajo inevitablemente obligarán a algún tipo de coordinación de las políticas entre sus miembros.

De esta forma, un papel más activo de APEC podría ser útil para dar respuestas oportunas sobre políticas y para una estrategia común sobre temas económicos relevantes de interés regional, considerando los desequilibrios comerciales, tasas de cambio, seguridad del abastecimiento de energía, ajuste estructural para la conservación ambiental y la política de inversión, todo ellos con el fin de preparar a las economías regionales para realizar ajustes, a tiempo, y disminuir los problemas potenciales.

\section{6.- Chile y APEC}

Como se sabe, Chile ingresó al APEC como miembro pleno durante la Reunión Ministerial de Jakarta, Indonesia, en noviembre de 1994 tras permanecer un año como invitado y participar en los grupos de trabajo ${ }^{43}$. La participación de Chile en APEC responde a los objetivos de la política comercial del país y es compatible con el principio de "regionalismo abierto", que inspira a la política de integración chilena ${ }^{44}$.

\footnotetext{
${ }^{41}$ Ver: Dirección Asia-Pacífico, Ministerio de Relaciones Exteriores. Relaciones Políticas y Económicas Bilaterales. Sitio web del Ministerio.

${ }^{12}$ Ver: BORTHWICK, Mark. Pacific Century: The Emergence of Modern Pacific Asia. Westview Press, Sydney, 1998.

${ }^{43}$ Ver: PEREZ, Marisol, Política Exterior de Chile hacia el Asia-Pacifico. En Chile-Japón, un Siglo de Amistad. Comisión Chilena de Celebración del Centenario de las Relaciones Chile-Japón. Santiago, 1997.

Dirección Asia-Pacifico, Ministerio de Relaciones Exteriores. Participación de Chile en los Organismos de Cooperación Transpacifica. Sitio web del Ministerio. El ingreso a este foro también estuvo en gran medida asociada al creciente comercio que Chile desarrolla con el resto de los miembros del grupo, que ha representado durante buena parte de la década de los noventa alrededor del $50 \%$ del intercambio con el mundo. A esto se
} 
Desde la perspectiva económica, el intercambio comercial entre Chile y los países asiáticos miembros de APEC, alcanzó durante el año 2000, 8.312 millones de dólares, con un saldo favorable para Chile en la balanza comercial de 2.312 millones. Las exportaciones de Chile a APEC totalizaron el año 2000 a 5.312 millones de dólares (FOB), representando un $28 \%$ del total exportado por Chile, siendo Japón el destino principal con 2.548 millones de dólares ${ }^{45}$

Las exportaciones de Chile son intensivas en el uso de recursos naturales y contienen poco valor agregado. Es ampliamente conocido el hecho de que las ventas nacionales se concentran mayoritariamente en materias primas como cobre, fruta, harina de pescado, celulosa, salmones, chips de madera y otras variedades forestales. Tercero, aunque el crecimiento de las exportaciones ha sido muy fuerte, $y$ aunque ha fomentado la expansión de envíos de productos no tradicionales, el universo empresarial chileno involucrado en el intercambio es aún pequeño ${ }^{46}$.

Por otra parte, la participación de los empresarios chilenos en el Consejo Económico de la Cuenca del Pacífico (PBEC), organismos que agrupa al sector privado, no está a la altura de nuestros vínculos comerciales, reflejando de esta el déficit existente en términos de la integración de pequeños y medianos empresarios a las posibilidades de intercambio que ofrecen las economías del Este Asiático. En consecuencia, Chile enfrenta ahora el desafío de incrementar y mejorar el perfil de sus exportaciones, integrando más conocimiento y mano de obra a los productos naturales que exporta ${ }^{47}$.

Ahora bien, la sola circunstancia de integrar el APEC le permite a Chile extender su acceso a los mercados y a las fuentes de inversión en el Pacífico en condiciones que difícilmente serían logradas a través de negociaciones individuales con cada una de las restantes 17 economías. Esta membresía en APEC le confiere a Chile un interesante espacio de maniobra para acelerar la apertura comercial bilateral con países como Nueva Zelandia y Malasia con el fin de consolidar una inserción múltiple y autónoma en el Este Asiático

agrega que gran parte de los principales inversionistas extranjeros en Chile son también miembros del APEC (Estados Unidos, Canadá, Australia y Japón).

${ }^{45}$ MUÑOZ, Heraldo. Chile y el Asia-Pacífico: Una Vieja Amistad para un Nuevo Milenio. En Diplomacia. N 88 , Julio-Septiembre 2001, Santiago. Página 24.

${ }^{46}$ Ver: SUBERCASEAUX, Enrique. Chile y APEC 2004: Desafios y Oportunidades ante Escenarios Cambiantes. En Diplomacia. $N^{\circ} 88$, Julio-Septiembre 2001, Santiago.

Ministerio de Relaciones exteriores. Documento de trabajo interno, sin fecha.

${ }^{48}$ Ver: PEREZ, Marisol. Política Exterior de Chile hacia el Asia-Pacifico. En Chile-Japón, un Siglo de Amistad. Comisión Chilena de Celebración del Centenario de las Relaciones Chile-Japón. Santiago, 1997. 
Desde la incorporación chilena al APEC, el norte de la diplomacia económica ha sido diversificar y ampliar el acceso a los mercado asiáticos, mejorar la calidad de nuestro intercambio, fomentar la creación de proyectos conjuntos y atraer inversión extranjera. La profundización de los lazos económicos con Asia-Pacífico, sin embargo, es sólo una dimensión de la nueva era, por cuanto el desafío de más proyección en la próxima etapa es sentar las bases para que el país se convierta en una 'plataforma estraté gica' o 'puerta' entre el Cono Sur latinoamericano y el Pacífico Asiático ${ }^{49}$.

De cualquier forma, como ha señalado Heraldo Muñoz, la relación económica con los paises del Asia-Pacífico está fundamentalmente basada en el comercio. Las inversiones directas, los flujos tecnológicos, la cooperación económica y política y el intercambio cultural, todavía se encuentran en niveles incipientes ${ }^{50}$.

Desde una perspectiva más especifica, también debe considerarse la relevancia que tiene para Chile el contar con la sede y la presidencia de un grupo experto de APEC: El Grupo Experto en Minería, Exploración y Desarrollo Energético, GEMEED, ${ }^{51}$ presidido desde su fundación por Tomás Astorga ${ }^{52}$.

${ }^{49}$ Ver: SUBERCASEAUX, Enrique. Chile y APEC 2004: Desafíos y Oportunidades ante Escenarios Cambiantes. En Diplomacia. N 88, Julio-Septiembre 2001. Santiago.

${ }^{50}$ MUÑOZ, Heraldo. Chile y el Asia-Pacifico: Una Vieja Amistad para un Nuevo Milenio. En Diplomacia. Nº 88 , Julio-Septiembre 2001, Santiago. De esta forma, Muñoz señala que subsisten problemas que afectan el comercio de los productos chilenos en esa zona: barreras no arancelarias, controles de importación, restricciones sanitarias y fitosanitarias, cuotas, tarifas discriminatorias y subsidios, además de las obvias dificultades de transporte y comunicaciones.

Ver: CAMPUSANO, Raul F. The Group of Experts on Minerals and Energy Development and its Role Regarding Copper Mining Sustainability in the Asia Pacific Region. Coautor: Tomás Astorga. Proceedings of Copper 99. Phoenix, Arizona, 1999.

${ }^{52}$ El año 1996 se constituye el Grupo Experto en Mineria, Exploración y Desarrollo Energé tico de APEC, GEMEED. Esta organización depende directamente del Grupo de Trabajo de Energía de APEC y cuenta con la activa participación de representantes de los gobiernos de Australia, Brunei, Canadá, Chile, China, China Hong-Kong, Indonesia, Japón, Corea, Malasia, México, Nueva Zelanda, Papua Nueva Guinea, Perú, Filipinas, Rusia, Singapur, Taipei Chino, Tailandia, Estados Unidos y Vietnam. La misión del Grupo Experto es la siguiente: Articular, coordinar y promover, dentro de APEC, materias relacionadas con la minería, intercambiando información asociada con la oferta de minerales y su exploración; Establecer nuevos tópicos dentro del marco del desarrollo sustentable, promoviendo la aplicación de nuevas tecnologías y medidas que mitiguen o eliminen los impactos sociales, económicos y ambientales negativos; Promover el adecuado acceso a mercados de los productos mineros; Establecer relaciones con organizaciones internacionales y con el sector de negocios relacionados con la minería en orden de aunar esfuerzos y lograr sinergias que fortalezcan la sustentabilidad de la actividad minera. Desde su fundación, GEMEED ha sido liderado por un presidente chileno y el Secretariado de la organización se encuentra en Chile, formando parte de un programa del Ministerio de Minería. GEMEED ha realizado una gran cantidad de actividades dentro de sus pocos años de existencia, entre las cuales cabe destacar las siguientes: Seis reuniones plenarias (Santiago, Seút, Ottawa, Bangkok, Las Vegas y Acapulco); Cuatro talleres de Cooperación Ambiental (Tokio, Santiago, Cairns, Acapulco); Promoción y publicación de estudios relacionados con minería y la región Asia-Pacífico; Mantención de un sitio web con amplia información sobre minería en la región; Ejecución de dos reuniones virtuales a través de Internet; Creación de una red de trabajo en todos los países de la región, la que ha permitido intercambio de información, apoyos recíprocos, sistema de alerta temprana frente a problemas relacionados con la mineria, mayor conocimiento mutuo, creación de confianzas, etc. 


\section{7.- Inversión extranjera en economías de APEC}

La inversión extranjera directa ha jugado un rol fundamental en el desarrollo económico de la región del Asia-Pacífico. Iniciada en forma significativa en la década de los 50 y continuando a los 70, la inversión extranjera en Asia estuvo marcadamente dominada por los Estados Unidos y Europa.

Las grandes multinacionales miraban a los países asiáticos, con su abundante y poco costosa mano de obra, como una ubicación privilegiada para la instalación de grandes industrias de montaje y manufactura. Una muestra bastante significativa del dominio occidental de la inversión extranjera en la región es el caso de Hong Kong: al iniciarse la década de los 70 , más de tres cuartos de la inversión extranjera directa provenían de Estados Unidos y Europa.

Sin embargo, ya a mediados de los 70 se puede observar un cambio gradual en los patrones de inversión. Los flujos intra-regionales de capital comienzan a crecer sustancialmente, con la consecuente mayor independencia respecto de las inversiones europeas y estadounidenses. Continuando con el ejemplo de Hong Kong, a inicios de la década de los 80 el monto de la inversión extranjera de occidente se había reducido al $60 \%$ y en 1989 era inferior al $47 \%$.

La situación anteriormente descrita no significa que la influencia de las economías occidentales en el desarrollo asiático esté desapareciendo, sino que los flujos de capital intra-regionales tendrán, desde la década de los 90 en adelante, un rol mucho mayor que el que tuvieron en el pasado.

La mayor fuerza detrás de este aumento intra-regional del flujo de capitales en el Asia ha sido el Japón ${ }^{55}$. Japón inició su primer gran empuje inversionista en el Asia a fines de los sesenta, como resultado de dos factores: el primero de ellos, relativo a los menores costos de producción de los demás países asiáticos con relación a la economía japonesa; y el segundo, relativo al hecho de que algunos de los países asiáticos a los cuales Japón exportaba sus productos establecieron restricciones a la importación, lo que trajo como consecuencia que producir en tales países fuese más provechoso.

Mientras la inversión japonesa en los países asiáticos continuaba en forma sostenida, el año 1985, como resultado de una significativa subida del Yen en los mercados internacionales, hubo un gran boom para las inversiones japonesas en el

\footnotetext{
${ }^{53}$ BORA, Bijit and Mari Pangestu, Eds. Priority Issues in Trade and Investment liberalization: Implications for the Asia-Pacific Region. Pacific Economic Cooperation Council, PECC. Singapore, 1996.

${ }^{54}$ Ministerio de Relaciones Exteriores. Documento de trabajo interno, sin fecha.

${ }^{55}$ Ver: SMITH, Patrick. Japan, a Reinterpretation. Vintage Books, New York, 1997.
} 
exterior. Entre 1987 y 1990, Japón invirtió más de 16 billones de dólares en las economías de Hong Kong, Singapur, Malasia, Tailandia, Indonesia, y Filipinas ${ }^{56}$.

Un caso específico del aumento de la presencia japonesa puede encontrarse en Tailandia: a inicios de los 80 la inversión estadounidense era el doble que la japonesa; en 1989, las inversiones japonesas representaban el 50\% de la inversión extranjera realizada en Tailandia, mientras que las inversiones estadounidenses representaban sólo el $14 \%{ }^{57}$.

Esta arremetida japonesa marcó el inicio de lo que podría ser llamado el "fenómeno de migración manufacturera", fenómeno que continúa dándose hasta el día de hoy. Al iniciarse el proceso, la mayor parte de la migración manufacturera se dio por parte de las compañías japonesas hacia ciertos países asiáticos que comparativamente permitían producir con menores costos.

En la década de los ochenta, las economías de tales países se habían desarrollado de tal manera, que los costos de producción aumentaron a un nivel que ya no los hacía convenientes como centros de manufactura; con lo cual, ya no sólo las compañias japonesas sino además muchas compañías de esos mismos países, comenzaron a buscar nuevos lugares de producción ${ }^{58}$.

Así, en 1990, Taiwan sobrepasó significativamente a Japón como el principal inversionista en Malasia, invirtiendo más de 2.3 billones de dólares. Hong Kong por su parte, se ha transformado en el mayor inversionista en China, con más de dos tercios de la inversión extranjera a su haber.

Singapur ha tenido un activo rol de inversionista en las economías de Malasia e Indonesia. Todos estos ejemplos nos muestran que, a pasar de seguir teniendo Japón un rol dominante en el desarrollo de la inversión extranjera al interior de las economías asiáticas, muchos otros países de la región están comenzando a tener un impacto en el flujo intra-regional de las inversiones $\mathrm{y}$, sin duda, tendrán un activo papel en el futuro próximo ${ }^{59}$.

Además del fenómeno anteriormente referido, la relajación de las tensiones políticas y económicas existentes entre algunos países asiáticos ha sido otro de los

\footnotetext{
${ }^{56}$ Ver: BORTHWICK, Mark. Pacific Century: The Emergence of Modern Pacific Asia. Westview Press, Sydney, 1998

"Ver: SMITH, Patrick. Japan, a Reinterpretation. Vintage Books, New York, 1997.

${ }^{58}$ BORA, Bijit and Mari Pangestu, Eds. Priority Issues in Trade and Investment liberalization: Implications for the Asia-Pacific Region. Pacific Economic Cooperation Council, PECC. Singapore, 1996.

Ver por contexto: TRESSLER, Frank. Banco de Desarrollo del Asia: un Sistema de Cooperación Regional para los Nuevos Tiempos. En Diplomacia. N 88, Julio-Septiembre 2001, Santiago.
} 
factores que ha favorecido el flujo de capitales a nivel intra-regional. Así, los agentes económicos de los diferentes países de la región han ido adquiriendo más confianza entre si y desarrollando mayores vínculos comerciales.

El mérito de este tipo de cambios es de muchos gobiernos de la región, que se han centrado en crear y desarrollar economías de mercado favoreciendo el intercambio comercial con los países vecinos. De esta manera, se han ido reduciendo las barreras económicas al interior de la región para permitir y favorecer la cooperación económica, fortalecié ndose asi la interdependencia de las economías del Asia-Pacífico ${ }^{60}$.

En resumen, Asia se encuentra en una importante etapa de transición: creciendo, de ser un centro de mano de obra barata, a transformarse en un mercado desarrollado y relativamente maduro.

Así, durante la década de los 90 la dependencia asiática de los flujos de capital de Estados Unidos y Europa ha ido declinando gradualmente y sus propias economías han sido capaces de ir generando los recursos, la producción y la demanda requeridos para alimentar el crecimiento de la economía regional. Asia se está preparando para emerger como una región autosuficiente y económicamente independiente ${ }^{61}$.

\section{8.- Chile-APEC 2004}

Durante todo el año 2004, Chile será anfitrión de las actividades del Foro de Cooperación Económica del Asia-Pacífico (APEC). Se abre así una oportunidad histórica de participación y protagonismo regional desconocida por Chile hasta la fecha. En efecto, como ha señalado Mario I. Artaza, el año APEC Chile 2004 será el evento internacional de mayor envergadura jamás organizado en nuestro país. Por primera vez en nuestra historia, durante la Cumbre Informal de líderes económicos APEC, los líderes de tres potencias nucleares, de tres miembros del G-8, de siete integrantes de ASEAN y de tres miembros del Grupo de Río, entre otras Economías APEC, estarán reunidos para discutir los avances que esta organización multilateral de cooperación habrá cumplido, en el umbral de su ambiciosa meta para reducir las barreras del comercio y la inversión para un mercado compuesto por más de un tercio de la población total de nuestro planeta ${ }^{62}$.

\footnotetext{
${ }^{60}$ Ministerio de Relaciones Exteriores. Documento de trabajo interno, sin fecha.

${ }^{61}$ BORA, Bijit and Mari Pangestu, Eds. Priority Issues in Trade and Investment liberalization: Implications for the Asia-Pacific Region. Pacific Economic Cooperation Council, PECC. Singapore, 1996.

ARTAZA, Mario I. Chile en APEC: Aprovechando lo mejor de la Globalización. Documento de trabajo, Singapur, 2001.
} 
Como se ha señalado, la región Asia-Pacífico es la más dinámica del planeta desde la perspectiva de su crecimiento económico, apertura de mercados, desarrollo de la minería y avances en libre comercio. ${ }^{63}$ No parece caber dudas respecto de la importancia y potencialidades que para Chile representa su participación protagónica en esta región. La región representó en el año 2000 el 54.3\% de las ventas al exterior de Chile ${ }^{64}$.

Ahora bien, ser anfitriones APEC el año 2004 no es sólo una oportunidad y un honor, sino que también es una responsabilidad, la que si no es asumida como corresponde, puede transformar ese año no sólo en otra oportunidad desaprovechada, sino también en descrédito como país ${ }^{65}$.

No teniendo en nuestra experiencia nacional eventos de la magnitud del señalado, todo indica que su preparación debe transformarse en un esfuerzo país y en una prioridad nacional. Entre los múltiples aspectos que deben considerarse, destacan los siguientes ámbitos generales:

\section{Aspectos presupuestarios.}

$>$ Recursos humanos.

$>$ Aspectos logísticos.

Materias substantivas que se desea presentar y discutir.

Sin embargo, a dos años del 2004, ninguno de los ámbitos señalados parece estar siendo abordado en forma adecuada. Debe considerarse que la experiencia comparada muestra a otras Economías de APEC, que prepararon una situación similar con al menos tres años de antelación. $Y$ efectivamente, son tantos los aspectos que deben tomarse en cuenta que dos años sean tal vez el plazo mínimo para organizar los eventos del año $2004^{66}$.

${ }^{63}$ CAMPUSANO, Raul F. The APEC Expert Group on Mining, Exploration and Energy Development, and its Commitment with the Environment. Proceedings of APEC-GEMEED. Taller de Cooperación Ambiental ECOW'98. Ministerio de Minería de Chile. Santiago, 1998.

${ }^{64}$ Ver: SUBERCASEAUX, Enrique. Chile y APEC 2004: Desafíos y Oportunidades ante Escenarios Cambiantes. En Diplomacia. No 88, Julio-Septiembre 2001, Santiago.

${ }^{65}$ Ver: PEREZ, Marisol. Política Exterior de Chile hacia el Asia-Pacífico. En Chile-Japón, un Siglo de Amistad. Comisión Chilena de Celebración del Centenario de las Relaciones Chile-Japón. Santiago, 1997

${ }^{66}$ Ver: ARTAZA, Mario Ignacio. Chile en APEC: Aprovechando lo mejor de la Globalización. En Diplomacia. N ${ }^{\circ}$ 88, Julio-Septiembre 2001, Santiago. También ver: SUBERCASEAUX, Enrique. Chile y APEC 2004: Desafios y Oportunidades ante Escenarios Cambiantes. En Diplomacia. N88, Julio-Septiembre 2001, Santiago. 
En relación con aspectos presupuestarios, todo parece mostrar que los recursos normales de los ministerios y demás servicios públicos son absolutamente insuficientes para financiar los gastos fundamentales del año 2004. Como ha señalado Artaza, si utilizamos como ejemplo los montos financieros que han requerido para enfrentar este desafío nacional en términos logísticos, de infraestructura, personal, alimentación y transporte, entre otros costos fijos, los gobiernos de Nueva Zelandia (Año APEC 1999), Brunei Darussalam (2000) y la república Popular China (2001), ${ }_{68}^{67}$ estamos hablando de un cifra en el rango de los US \$15 a US \$2 27 millones $^{68}$.

En relación con recursos humanos, éste es tal vez el aspecto de mayor debilidad. A pesar del espíritu, entusiasmo y enorme esfuerzo de los dos funcionarios de Cancillería en Santiago y del funcionario de Cancillería en Singapur, ciertamente la dotación es insuficiente para enfrentar el trabajo. Si se observa la situación en otros ministerios y servicios públicos la situación es aún más desoladora. En la mayoría de los casos, la reunión del 2004 y en general APEC simplemente no es un tema. En otros casos, existe un funcionario aislado que trabaja y se esfuerza más bien por motivación propia que por una decisión y política institucional. Por otra parte, la apertura al sector privado (sector empresarial, académico, sociedad civil y público en general) es mínima y casi inexistente. De hecho, la mayoría de los ciudadanos no sospecha que Chile será anfitrión de APEC el año 2004. No hay difusión y no parece haber planes concretos hacia delante.

De la misma forma, la experiencia comparada parece demostrar la necesidad de abrir la materia en todos sus aspectos a la ciudadanía e integrarla como protagonista del evento, dejando de lado la tendencia a trabajar a puertas cerradas desde la desconfianza a la sociedad civil $^{69}$.

En relación con aspectos logísticos, la realidad de nuestro país deja mucho que desear. Simplemente, Chile no está preparado hoy para recibir y atender a todas las

\footnotetext{
${ }^{67}$ TORO, Sergio. 2001 Annus Admirabilis: La Política Exterior de China de la Guerra del Opio a la Globalización. En Diplomacia. N 88 , Julio-Septiembre 2001, Santiago.

${ }^{68}$ Artaza, op. cit.

${ }^{69}$ En esta línea, es ilustrativo citar a Artaza, que ha señalado que hoy existe un creciente grado de temor entre los personeros de varios gobiernos para tratar los temas vinculados al proceso de globalización de una manera abierta, franca y constructiva, con la mayor participación posible de la ciudadanía, lo que para Chile, a tres años de organizar su año APEC 2004, es también un aliciente para iniciar desde ya un proceso de información y de educación en la comunidad, promoviendo la participación y el sentido de pertenencia del éxito de este evento multilateral internacional que se realizará en Chile, diferenciándose asi de algunas normas que están siendo incorporadas en las agendas gubernamentales de varias naciones del planeta. Estas incluyen el alejar las críticas del proceso de debate, aunque sean éstas constructivas; decidir sobre la base de lo que plantean los representantes de las multinacionales, la banca internacional, el mercado accionario o ciertos "especialistas" que forman parte del establishment político de turno; informar, a quienes serán los más afectados por la implementación de ciertas medidas de apertura comercial, lo esencial, lo mínimo, en un lenguaje que no entienden. Op. Cit, páginas 95 y 96.
} 
personas que vendrán el año 2004. Se habla de al menos 4 ciudades que deben prepararse para desarrollar eventos asociados.

En relación con materias substantivas que se desea presentar y discutir, parece evidente que ésta es una gran oportunidad para influir en la agenda APEC y para mostrar al país y desarrollar oportunidades de negocios, cooperación, intercambios, etc. Por otra parte, no pareciera ser aconsejable dispersar el esfuerzo en muchas materias y sectores. Por el contrario, pereciera adecuado focalizar en dos o tres temas y desarrollarlos consistentemente.

Uno de esos temas puede ser la minería. Existen diversas razones para ello, entre otras las siguientes:

$>$ Es un sector en el que somos líderes mundiales y tenemos mucho que mostrar, desde nuestros proyectos con tecnologías de punta a nuestros recursos humanos de primer nivel y que pueden desempeñarse exitosamente en otros entornos, hasta nuestros problemas ambientales y sociales y cómo estamos enfrentándolos y resolviéndolos.

$>$ El trabajo del sector minero en APEC data del ingreso de Chile a la organización y se consolida el año 1996, con la creación del Grupo Experto en Minería, Exploración y Desarrollo Energético, GEMEED-APEC. ${ }^{70}$ Cómo se sabe, es el único grupo experto de APEC cuya secretaría y presidencia están radicadas en Chile. Más aún, desde su creación, el Grupo ha organizado y realizado exitosamente reuniones plenarias anuales a ambos lados del Océano Pacífico, como también talleres internacionales de cooperación ambiental y otros. También ha llevado a efecto reuniones virtuales y publicado varios textos. GEMEED es bien conocido en el sector minero de las Economías de APEC y cuenta con una red de delegados, contactos, colaboradores y amigos ya consolidada.

\footnotetext{
${ }^{70}$

${ }^{70}$ Ver: CAMPUSANO, Raul F. The Group of Experts on Minerals and Energy Development and its Role Regarding Copper Mining Sustainability in the Asia Pacific Region. Coautor: Tomás Astorga. Proceedings of Copper 99. Phoenix, Arizona, 1999. También ver: CAMPUSANO, Raul F. The APEC Expert Group on Mining, Exploration and Energy Development, and its Commitment with the Environment. Proceedings of APEC-GEMEED. Taller de Cooperación Ambiental ECOW' 98. Ministerio de Minería de Chile. Santiago, 1998.

7

La labor de GEMEED ha sido amplia y fructífera. Esto nos ha permitido un posicionamiento como país inédito en otros foros y sectores, abriendo posibilidades de generación e implementación de políticas en el sector minero inimaginables de otra forma, $Y$ sin embargo, hay veces en que pareciera que las autoridades no comprendieran el alcance y las potencialidades de la región en general y de GEMEED en particular. En efecto, la política del pais de privilegiar todos los foros, todas las regiones y todos los posibles acuerdos comerciales con todos los paises que estén dispuestos a negociar con Chile (y que a veces ha sido denominada como "regionalismo abierto") es en realidad una forma de no privilegiar relación alguna. Cada vez que aparece una oferta nueva de negociación de tratado de libre comercio todas las fuerzas de la administración se vuelcan hacia allá, dejando en el aire trabajo de años y provocando gran confusión y sorpresas en nuestros socios tradicionales, particularmente aquellos de la
} 
El sector minero de Chile tiene la voluntad de participar activamente en las actividades del año 2004. Así lo demostró el Taller de Planificación estraté gica de GEMEED realizado recientemente en las premisas del CIMM, en que la presencia de la Subsecretaria sectorial, de los tres jefes de Servicio del sector, del Gerente General del Consejo Minero, de un alto representante de SONAMI, del Presidente del Instituto de Ingenieros de Minas, y de otras autoridades y representantes del sector público y privado, dan testimonio del compromiso del sector con la región Asia-Pacífico.

Estamos a tiempo para enfrentar exitosamente el desafío del 2004, pero debemos lograr que el país en su conjunto asuma este desafío y oportunidad y ponernos a trabajar ya.

\section{9.- Referencias}

1. APEC Business Advisory Council. Report to APEC Economic Leaders. Shanghai, 2001.

2. APEC Senior Officials. 2001 Report on Economic and Technical Cooperation. Asia-pacific Economic Cooperation. Singapore, 2001.

3. ARTAZA ROUXEL, Mario. El Foro de Cooperación América Latina-Asia del Este. En Diplomacia. № 88, Julio-Septiembre 2001, Santiago.

4. ARTAZA, Mario Ignacio. Chile en APEC: Aprovechando lo mejor de la Globalización. En Diplomacia. N 88, Julio-Septiembre 2001, Santiago.

5. BORA, Bijit and MARI PANGESTU, Eds. Priority Issues in Trade and Investment liberalization: Implications for the Asia-Pacific Region. Pacific Economic Cooperation Council, PECC. Singapore, 1996.

6. BORTHWICK, Mark. Pacific Century: The Emergence of Modern Pacific Asia. Westview Press, Sydney, 1998.

ribera asiática de la Cuenca del Pacífico, que valoran las relaciones estables y claras en gran medida. Es cierto que el Ministerio de Minería ha hecho posible la participación de nuestro pais en GEMEED y que la Comisión Chilena del Cobre ha desarrollado una brillante participación nacional en los foros internacionales de GEMEED. Pero también lo es que la organización no cuenta con todo el respaldo y apoyo que sería menester. En efecto, el presupuesto anual de GEMEED es muy pequeño para el cumplimiento de su programa de trabajo, cada año es incierto que el presupuesto anual será aprobado, hay una serie de trabas y complicaciones administrativas, hay quienes no comprenden la relevancia del tema y las posibilidades y proyecciones abre para el país y su minería, etc. Más aún, si la burocratización, incapacidad de toma de decisiones y señales contradictorias se mantienen, Chile corre el peligro de perder credibilidad frente a sus contrapartes de la región perdiendo una oportunidad privilegiada que se logró con gran esfuerzo, tenacidad y perseverancia. 
7. BURSTEIN, Daniel y Arne de Keijzer. Big Dragon: China's Future, what it means for Business, the Economy, and the Global Order. New York, 1998.

8. CAMPUSANO, Raúl F. Estudio Comparado de Ordenamientos Jurídicos en Economías APEC: Derecho del Medio Ambiente y la Actividad Minera en Australia, Canadá, China y Japón. Colaboradora: Emilia Bolocco. Ministerio de Minería, 1999.

9. CAMPUSANO, Raúl F. Copper, Market Growth Potential and Threats in the Asia Pacific Region. Coautor: Tomás Astorga. Proceedings of Copper 99. Phoenix, Arizona, 1999.

10. CAMPUSANO, Raúl F., The Group of Experts on Minerals and Energy Development and its Role Regarding Copper Mining Sustainability in the Asia Pacific Region. Coautor: Tomás Astorga. Proceedings of Copper 99. Phoenix, Arizona, 1999

11. CAMPUSANO, Raúl F., The APEC Expert Group on Mining, Exploration and Energy Development, and its Commitment with the Environment. Proceedings of APEC-GEMEED. Taller de Cooperación Ambiental ECOW'98. Ministerio de Minería de Chile. Santiago, 1998.

12. Comité Empresarial Chile-Japón. Actas de la 19a Reunión del Comité Empresarial Chile-Japón. Tokio, 1999.

13. CHU, Chin-Ning. The Asian Game. Stealth Productions Australia. St. Ives, 1995.

14. Dirección Asia-Pacífico, Ministerio de Relaciones Exteriores. Foro América Latina-Asia del Este (FALAE). Sitio web del Ministerio.

15. Dirección Asia-Pacífico, Ministerio de Relaciones Exteriores. Participación de Chile en los Organismos de Cooperación Transpacífica. Sitio web del Ministerio.

16. Dirección Asia-Pacífico, Ministerio de Relaciones Exteriores. Relaciones Políticas y Económicas Bilaterales. Sitio web del Ministerio.

17. DOBBS-HIGGINSON, M. S. Asia-Pacific, its Role in the World Disorder. The Japan Times. Tokyo, 1993.

18. E-APEC Task Force. E-APEC Strategy 2001. Asia-Pacific Economic Cooperation, Singapore, 2001. 
19. GUTIERREZ, Hernán. Chile y Japón: Hacia una Asociación Estratégica. En Diplomacia. N 88, Julio-Septiembre 2001, Santiago.

20. Meeting of APEC Ministers Responsible for Trade. Key APEC Documents 2001. Asia-Pacific Economic Cooperation, Singapore, December 2001.

21. MUÑOZ, Heraldo. Chile y el Asia-Pacífico: Una Vieja Amistad para un Nuevo Milenio. En Diplomacia. Nº8, Julio-Septiembre 2001, Santiago.

22. Osaka Actino Agenda: 2001 Update. Key APEC Documents 2001, AsiaPacific Economic Cooperation, Singapore, December 2001.

23. PÉREZ, Marisol. Política Exterior de Chile hacia el Asia-Pacífico. En ChileJapón, un Siglo de Amistad. Comisión Chilena de Celebración del Centenario de las Relaciones Chile-Japón. Santiago, 1997.

24. Shanghai Accord: Meeting New Challenges in the New Century. APEC Leaders Declaration. Shanghai, 2001. Key APEC Documents 2001, AsiaPacific Economic Cooperation, Singapore, December 2001.

25. SMITH, Patrick. Japan, a Reinterpretation. Vintage Books, New York, 1997.

26. SUBERCASEAUX, Enrique. Chile y APEC 2004: Desafíos y Oportunidades ante Escenarios Cambiantes. En Diplomacia. N 88, Julio-Septiembre 2001, Santiago.

27. TORO, Sergio. 2001 Annus Admirabilis: La Política Exterior de China de la Guerra del Opio a la Globalización. En Diplomacia. N 88, Julio-Septiembre 2001, Santiago.

28. TRESSLER, Frank. Banco de Desarrollo del Asia: un Sistema de Cooperación Regional para los Nuevos Tiempos. En Diplomacia. N 88, Julio-Septiembre 2001, Santiago.

29. WILHELMY, Manfred. El proceso de Reformas en China y su Política Exterior. Estudios Públicos, Centro de Estudios Públicos, N78, 2000. 